Journal of Social Sciences 2 (1): 14-20, 2006

ISSN 1549-3652

(C) 2006 Science Publications

\title{
Multi-activity and Brand Name Local Products on an Island of the North Aegean, Greece
}

\author{
${ }^{1}$ Fotis Chatzitheodoridis, ${ }^{2}$ Ioannis Mountousis, ${ }^{2}$ Konstantinos Papanikolaou and ${ }^{1}$ Dimitrios Tsilochristos \\ ${ }^{1}$ Faculty of Agricultural Technology, T.E.I. of Western Macedonia, Florina 53100 GR \\ ${ }^{2}$ Faculty of Agriculture, Aristotle University of Thessaloniki, Thessaloniki 54006 GR
}

\begin{abstract}
The present work refers to a research that took place on Limnos, an island found in North Aegean, the more northern islander region of Greek Archipelagos. In the frame of the research, the factors that shape and influence the rural growth of the island were studied. Via these factors, the structure of rural occupation, as well as the multi-activity phenomenon is described. Moreover, the forms of rural occupation are combined with the farmer's confidence and opinion concerning the brand name rural products of the island. From the results of this research it becomes clear that the phenomenon of rural multi-activity is particularly intense and is related to other occupations in the tertiary sector. The brand name rural local products are mostly supported by the exclusively and mainly occupied in primary sector people, while the multi-activated young people that are secondarily or auxiliary occupied in agriculture dispute the value of local brand name products and their contribution in the growth of the island.
\end{abstract}

Key words: Multi-activity, brand name local products, insular development, factor analysis

\section{INTRODUCTION}

The insular areas, mainly due to their structural characteristics and problems, are less developed compared to mainland regions. The particular characteristics of the islands are grouped in two categories. The first category is related to their small size that is identified with the existence of limited raw material and natural environment, with small sized market and with limited bearing capacity. The second category of characteristics is related to the remoteness and isolation of the islands, a fact that results in increased pecuniary cost of living, of acquisition of projects and infrastructure services and of opportunities ${ }^{[1]}$.

Most of the islands were influenced, as for their developmental prospect at the postwar period, from the explosion of tourist activity which became synonymous with the massiveness of the number and organization of tourists and was based on the model of heliotropismtourism, in other words on the projection and consumption of mainly three resources: the sun, the sea and the coasts. In this frame, the islands developed into main tourist destinations as they usually combine and offer these resources in abundance. The growth of tourism brought about important profits regarding employment and income in the islander economy, but also in many cases, it created and still creates environmental pressures and problems ${ }^{[2]}$.

In the frame of the European Union the islander areas present a relatively big primary sector, a very limited industrial sector and an oversized sector of services. The importance of primary sector regarding occupation is reflected in the big number of small exploitations or in the dependence on fishery. The reduction of agricultural income at the last decades forces the farmers either to abandon this work-field or to turn to secondary activities.

The growth policies of the European Union that concern and are applied in the mountainous and disadvantageous regions, in which many of the islands are included, mainly aim in the exploitation of local resources and products, in the management of the environment and cultural wealth, while they seek the rural income to be supplemented by a lot of occupations $^{[3]}$.

In the Greek islands, agriculture and cattlebreeding were and still are the basic activities of their residents. During the last decades, the importance of primary sector in the islander economy is continuously diminished, as the growth of tourism functions competitively towards human potential and the land. Farmer's multi-activity constitutes an extensive phenomenon in the islander regions and it is usually related to their main or secondary employment in the tertiary sector.

The multi-activity despite the many definitions that are met in the bibliography is more completely described as: "the combination of rural productive activity in familial exploitation with one or more other remunerated activities inside or outside the rural sector" ${ }^{\text {"[4] }}$. Initially the phenomenon of multi-activity concerned mainly the heads of rural exploitations, however at the last decades it is expanded to all the

Corresponding Author: Fotis Chatzitheodoridis, School of Agricultural Technology, T.E.I. of Western Macedonia, Florina $53100 \mathrm{GR}$ 
members of the rural household ${ }^{[1,3]}$. Due to the multiactivity, the head of the exploitation, histher spouse but also the remainder members of the household were in the present research considered as the carriers.

The growth causes of multi-activity are related to the farmer's need for acquisition of additional income, for guarantee against imponderable factors and to their effort to correspond to the presented high consuming models. The extent of multi-activity phenomenon fluctuates roughly in the $50 \%$ of total of rural households in the Greek reality ${ }^{[4,5]}$, while in the islander regions it exceeds this percentage many times. The multi-activity ended up to be a way of reproduction of the small and intermediate agricultural exploitation, a way of survival of certain mountainous and islander small societies and a way of environmental protection.

The structural characteristics of the islands, their high dependence on the occupation in the agricultural sector and on the growth of tourism, determine the need for "qualitative" and integrated growth. Towards this direction, apart from the multi-activity of the countryside, also pleads the exploitation of local goods or of services produced or provided in the countryside with the established socio-economic identity as the "powerful trump" of the regions ${ }^{[6]}$.

In the present research, the rural products regarded as local qualitative or brand name products are mainly those that incorporate name of origin and those that are established as such in the frame of the market. Two regulations referring to the labeling of such products (Regulations 2081/92 and 2082/92) exist in the Community legislation. The first concerns the protection of geographic indication and designation of origin, while the second the certification of particular characteristics. In the last few years an important increase in the demand and consumption of products that incorporate labeling of designation of origin is observed, while their price is higher and in certain cases double of the rest ${ }^{[7]}$.

Some of the agricultural and cattlebreeding exploitations in mountainous and disadvantageous regions, in which the islander are also included, are connected to the production of products which incorporate particular characteristics of geographic origin and are of superior quality. Specifically, from the 560 enacted products of Protected Designation of Origin (PDO) or Protected Geographical Indication (PGI) the 70\% derived from these regions of Europe ${ }^{[8]}$. These products usually slip the "productive logic" and approach "the logic of quality" $[9]$, while they also function as "an indication or a sign" of the region as they create positive associations for the place and the product to the consumers. The wine, for Greek islands as Samos, Limnos, Santorini or certain cheese products for islands as Lesvos and Limnos, constitute examples of such kind of products.
In this present research an effort to investigate the factors that compose and influence the rural occupation on the island of Limnos, which belongs to the disadvantageous regions of the European Union, takes place. Simultaneously, via these factors, the phenomenon of multi-activity as well as the relation of forms of occupation to the brand name rural products of the region is approached.

Study area: As a research region, selected was the island of Limnos, which is found in the centre of Northern Aegean and is the 8th bigger island of Greece. In the 20 years from 1981 to 2001 the population of the island increased at $10 \%$ (from 15.721 in 17.288 residents). The main sectors of occupation on Limnos are the services, the agriculture and the cattle-breeding. During the decade 1981 - 1991 there was an important change of occupations at the productive sectors. Specifically, there was a $38,8 \%$ decrease of primary sector and a 47,3\% increase of tertiary sector. At the same time, there was not any important change in the total of the cultivated extents and in the extensional structure of the cultures, while there was an extent increase in nine settlements of the island at the cost of pasture lands.

Limnos, during its history of many centuries, is connected with the production of products widely known for their particular characteristics and their origin. The "Limnia land", the "Limnio" wine, the "Kalathaki" cheese, the "Limnos" wheat and the cotton supported the local economy in various seasons and some of these do so until today.

During the '50s and the ' 60 s, due to the negative results of the culture of the brand name cotton that was produced on the island, the last big wave of immigration from Limnos to various countries abroad but also to the big urban centers of Greece was marked. The island and its local economy were literally denuded from the capable human potential and the survival of the people who remained was based on the agricultural production, on the transfers of the immigrants and on the presence of army. The inversion of the economic recession began from the ' 80 s and was mainly based on the local rural products and on the faintly developing tourism. Today, $90 \%$ of the produced quantities of local wines and cheeses of Limnos incorporate labeling of "Designation of Origin". They contribute in the income of the island's people who are engaged in vinicultures and cattle-breeding, while at the same time the quality of these products sets the proper conditions for future increase of their incomes.

Enacted with the Presidential Decree 243/1982, types of wines from Limnos under the label "Designation of Origin" are: the White dry wine by Designation of Origin of Superior Quality (D.O.S.Q.) with the indication "Limnos" and the dulcet wines, with the indication "Moschatos Limnos". The cheese 
"Kalathaki Limnos" is also legislated by the Ministerial Decision 313044 /1994 "Recognition of Protected Designation of Origin" and based on this it acquired the preferential labeling P.D.O. from the State.

Finally, the thyme honey of Limnos could be reported as a brand name local product, which even though it does not dispose labeling, it is sanctioned by the market because of its high demand and price as nearly the total of the produced quantity is sold directly to the big industries of process and standardization of honey.

Data sources and methods: In the research the main volume of primary data was assembled through the use of questionnaire addressed to the selected sample of population of the research's region. The farmer's questionnaire included questions that concerned in: the individual and family characteristics of the person asked, his employment, income, agricultural and cattlebreeding exploitations and his attitude and perception towards the local products and the environment.

As sampling technique the "stratified random sampling optimum allocation by Neyman" was selected. Based on that, the size of the sample was determined and was calculated in 240 rural households.

The most important methods of analysis that were used were the Factor Analysis, first and second degree, with the use of SPSS 12.0 (SPSS Inc 2003). The factor analysis is based on the admission that factors, which can be used in order to explain complex phenomena, do exist. The method substitutes a crowd of interdepended variables with a team of factors, hypothetical units, which behave in the same way as the statistically important variables that are contained in them. Each factor has its own identity and includes a team of variables connected to each other.

\section{RESULTS AND DISCUSSION}

From the research data related to the occupied in primary sector, it was found that percentage $45,8 \%$ (110 asked) of the sample is mainly occupied, allocating more time and acquiring higher income, in the primary, percentage $8,8 \%$ (21 asked) in the secondary and percentage $45,8 \%$ (109 asked) in the tertiary sector (Fig. 1).

Out of the 240 farmers that constituted the research's sample, the 181 (percentage 75,4\%) are also occupied in other kind of work except rural exploitation and the 59 (percentage 24,6\%) are completely and exclusively occupied in the primary sector's agricultural exploitation (Fig. 2).

Out of the 181 multi-activated farmers, the 156 (percentage $86,3 \%$ ) are supplementary occupied in primary and tertiary sector, the 15 (percentage 8,2\%) in primary and secondary, the 4 (percentage 2,2\%) in different kind of work in primary and the remainder 6

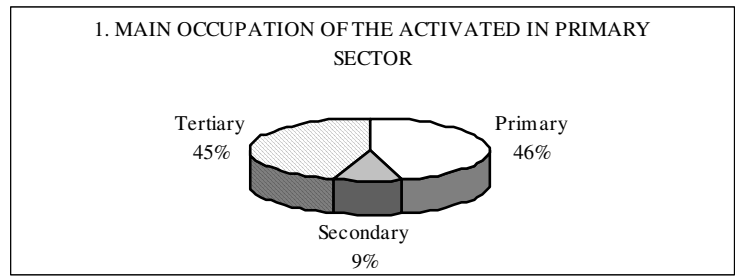

Fig. 1: Main occupation of the people activated in primary sector

2. PERCENTAGE OF THE EXCLUSIVELY AND MULTI-ACTIVATED IN PRIMARY SECTOR

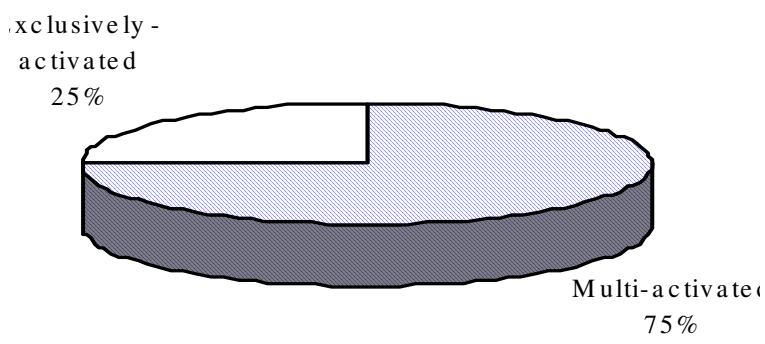

Fig. 2: Percentage of the exclusively-activated and multi-activated people in primary sector

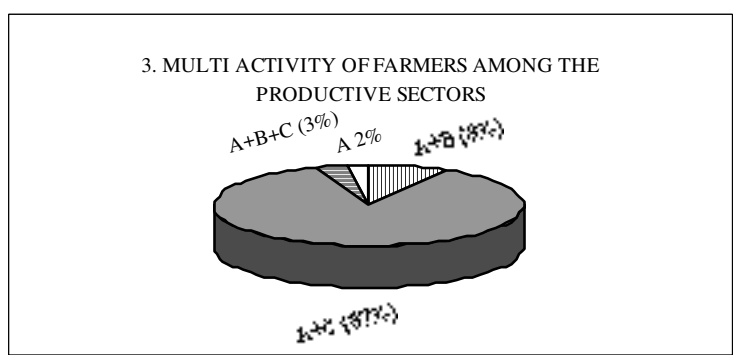

Fig. 3: Multi-activity of farmers among the productive sectors. (A=primary sector, $\mathrm{B}=$ secondary sector, $\mathrm{C}=$ tertiary sector)

(percentage $3,3 \%$ ) in all the three economic sectors (Fig. 3.)

In fact, we refer to rural multi-activity that is turned to the tertiary sector. The dependence of the agricultural households on incomes coming outside agriculture is appreciated, according to the above, to be $54 \%$.

This data led to the further investigation of the occupation and the phenomenon of multi-activity in correlation to the structure of rural exploitations but also to the attitude of farmers towards the rural qualitative products that are produced ad loc. These conditions and attitudes were broadened with the application of factor analysis of first degree, with rotation of biggest fluctuation in 18 variables that concerned in the individual characteristics, the exploitation characteristics and the sample's perceptions. The results that came out the application of this method are presented in Table 1. 
Table 1: Identification of factors based on the uterus of main components after rotation of biggest fluctuation

\begin{tabular}{|c|c|c|c|c|c|c|c|c|c|}
\hline \multirow{2}{*}{\multicolumn{2}{|c|}{$\begin{array}{l}\mathrm{P}_{2} \\
\text { Multi-activated with } \\
\text { secondary occupation } \\
\text { in primary sector }\end{array}$}} & \multirow{2}{*}{\multicolumn{2}{|c|}{$\begin{array}{l}\mathrm{P} 2_{2} \\
\text { Local Qualitative } \\
\text { Products }\end{array}$}} & \multirow{2}{*}{\multicolumn{2}{|c|}{$\begin{array}{l}\mathrm{P} 3_{2} \\
\text { Mainly occupied in } \\
\text { agriculture }\end{array}$}} & \multirow{2}{*}{\multicolumn{2}{|c|}{$\begin{array}{l}\mathrm{P} 4_{2} \\
\text { Young, multi-activated } \\
\text { occupied in agriculture }\end{array}$}} & \multirow{2}{*}{\multicolumn{2}{|c|}{$\begin{array}{l}\mathrm{P}_{2} \\
\text { Exclusively occupied in } \\
\text { Cattle-breeding }\end{array}$}} \\
\hline & & & & & & & & & \\
\hline Variab. & Burden & Variab. & Burden & Variab $^{2}$. & Burden & Variab. & Burden & Variab. & Burden \\
\hline MUAC & 0.89 & OPFU & 0.76 & AREA & -0.79 & AGE & -0.78 & ANEA & 0.78 \\
\hline MU13 & 0.83 & $\mathrm{OPCH}$ & 0.68 & AGEA & 0.76 & FPOS & 0.71 & OWEA & 0.63 \\
\hline ROM & 0.75 & OPWN & 0.64 & EDUC & -0.37 & EDUC & 0.52 & COOP & 0.31 \\
\hline ME1 & -0.73 & ORIG & 0.53 & OWEA & 0.45 & & & & \\
\hline ME3 & 0.70 & COOP & 0.49 & & & & & & \\
\hline INPU & 0.70 & & & & & & & & \\
\hline EDUC & 0.33 & & & & & & & & \\
\hline AGEA & -0.26 & & & & & & & & \\
\hline ANEA & -0.33 & & & & & & & & \\
\hline
\end{tabular}

* The name of the variables is presented in the Appendix Table.

1st factor (P1 $\mathbf{2})$ : "Multi-activated with secondary occupation in primary sector": Based on the crosscorrelation factors of variables with the factor "Multiactivated with secondary occupation in primary sector" the following is realized: Multi-activated are mainly the heads of rural households or their spouses, who are supplementary occupied in primary sector and are led to this second occupation as the income from the main employment, in most cases, is not enough to satisfy their needs. This multi-activity that they develop is composed of jobs that belong to primary and tertiary sector, while the total income they finally gain out of these jobs satisfies them. The multi-activated according to this first factor, have relatively high education and declare as their main occupation their work mostly in tertiary sector and as their secondary the one in primary sector. This fact is mainly connected to the small extent of cultivated land and the small extent of used pasture lands. In consequence, the income from these exploitations is supplementary and the time allocated in them is also less than the one allocated in their main employment $^{[10]}$.

This factor that also combines the biggest number of variables, describes the multi-activity giving the economic dimension as the main cause for the growth of the phenomenon. Specifically, the multi-activity is developed by:

The heads of the households and their spouses, that mainly inhabit the semi-urban centre of the island and work in the sector of services (employees, tradesmen, etc.). At the same time, they own relatively small exploitations that came to their property hereditary or were acquired. By the acquired exploitations, they aimed either to profit by the cashing of funds or to cultivate and produce products for themselves or products that attribute relatively ensured income with limited cost and care, e.g. grapes for wine-making. The role of the spouses, women mainly, is in this case to subserve and assist this familial effort. The main aim of this second occupation appears to be the supplementary income, but many times it is also the need to make use of spare time, the love for land and exercise, in combination with the culture for the production of "pure" products that supply their own alimentary needs.

The research showed that the specific multiactivated people are mainly activated in viniculture on the island of Limnos which leads to the production of brand name wines and secondarily in very small goatsheep-breeding exploitations, but also in land exploitations e.g. cereals, aiming at the funds given out for hard wheat production.

The final and total summed up income which emanates from the tertiary and primary sector, covers the needs of multi-activated households, as the relatively high education of the multi-activated helps them in the planning and process of the installation, the best utilization of their exploitation and the general economic planning of their household.

2nd factor P2 2 : "Local Qualitative Products": According to the data of the second factor, called "local qualitative products" it is realized that the exclusively or mainly occupied in primary sector and more specifically the ones who participate in rural cooperative societies:

Believe in and support the viniculture and therefore the produced wines because they consider them local products that have the potential and the dynamics to increase the vinicultures income but also to contribute in the economic growth of Limnos island. Despite the occasional crises in the viniculture-wine sector, related to the overproduction and creation of indisposed reserves, the release of trade and the worldwide competition increase also affecting the wine sector of Limnos (e.g. period 1992-94), the exclusively or mainly occupied in the rural sector people continue to entrust the particular activity and its final products. This confidence springs from the particular qualitative characteristics of the local wines, the space for their further exploitation and the sentimental relation that binds them. This fact is also verified by the planting of new vineyards, between 1995 and 2001 that increased the extent of the viniculture area of Limnos roughly at $15 \%$, while programs related to funds for the 
extirpation of vineyards had very poor results in the particular region.

This confidence is also highly confirmed in the case of the local cheese produced by goat and sheep milk and for which they consider that its most excellent quality and exclusive geographic origin constitute the main causes the particular product substantially contributes in the cattle-breeders income. In fact, the produced quantities of the cheese did not face problems of disposal or low price, as this product is widely known because of its qualitative characteristics and Designation of Origin that it incorporates, but also because of the limited quantities of production due to limited raw material.

Regarding the case of another qualitative rural product which does not incorporate labeling, but could become known as local qualitative product in specialized markets, they believe is the thyme honey of Limnos. Indeed, the thyme honey of Limnos has been "vouchsafed" via the market during the decades as its demand is particularly high and its annual production is almost entirely sold immediately to the relative industries of honey due to its particular characteristics and certainly due to the limited quantity produced. The thyme honey could have a wider "recognition" by selling it in "retail", which would make it more widely known, would make bigger profits for the producers and would set it as a local qualitative product of the island.

The exclusively or mainly occupied in rural sector who entrust and support the brand name local products, also accept or think of adopting a new culture, under the condition that they will maintain the income they gain from the already existing cultures and at the same time this energy will result in environmental profits for their place. The ominous prospects erased for the island's basic culture, that is the cereals, but potentially also the farmer's perception of their grounds degradation, mostly impose this attitude they have ${ }^{[11]}$.

3rd factor $\left(\mathbf{P 3}_{2}\right)$ : "Mainly occupied in Agriculture": Based on the cross-correlation factors of the variables with the factor "mainly occupied in agriculture", it is realized that the factor is referred to farmers who cultivate relatively big extents of agricultural land, live in regions where tourism is not developed, have relatively low education and are owners of big extents of rural land. It should be marked that as much as empirically speaking by the on the spot research, as with further investigation of the factor, it is realized that these holders of agricultural land mainly cultivate cereals, are household heads, participate in rural cooperative societies and their main employment is in primary sector.

4th factor $\left(\mathbf{P 4}_{2}\right)$ : "Young, multi-activated occupied in agriculture": According to the cross-correlation factors
Table 2: Identification of factors (secondary factor analysis) based on the uterus of main components after rotation of biggest fluctuation

\begin{tabular}{llll}
$\mathrm{P} 1.2$ & \multicolumn{3}{l}{$\mathrm{P} 2.2$} \\
\hline \multicolumn{2}{l}{ Multi-activity in primary sector } & \multicolumn{2}{l}{ Local rural products support } \\
\hline Variable $^{2}$ & Burden & Variable & Burden \\
$\mathrm{P}_{2}$ & 0.76 & $\mathrm{P} 2_{2}$ & 0.73 \\
$\mathrm{P} 4_{2}$ & 0.59 & $\mathrm{P}_{2}$ & 0.63 \\
$\mathrm{P} 3_{2}$ & 0.55 & $\mathrm{P} 3_{2}$ & -0.48 \\
\hline$*$ & The name of the variables is presented in the Appendix Table.
\end{tabular}

of the variables with the factor "Young, multi-activated occupied in agriculture" it is realized that these people are mainly family members, are young in age and of relatively high education. These individual characteristics lead mainly in second occupation in primary sector, an opinion strengthened by the relatively relaxed negative cross-correlation factor with the variable "mainly occupied in primary sector". In consequence, the young members of the families are mainly occupied in tertiary sector and supplementary in rural work.

In this case we substantially refer to young people secondarily occupied or occupied in agriculture who come as helpers or auxiliary hands in the familial effort, which usually starts from the head of the household and aims at the utilization of small exploitations for the acquisition of additional income. It is likely, that their "obligatory" relation to the familial exploitation and the social models they adopted at the duration of their studies in combination with their relatively young age, don't allow them to accept their agricultural occupation in a positive way. In consequence it is justifiable for them to be unsatisfied and to face the problems and the prospects of rural sector indifferently.

5th factor $\left(\mathbf{P 5}_{2}\right)$ : "Exclusively occupied in cattlebreeding": From the cross-correlation factors of the variables with the factor "exclusively occupied in cattle-breeding" it arises that they are farmers-breeders who use relatively big extents of pasture lands, are simultaneously owners of big extents of rural land and participate actively in rural co-operative societies.

As the research showed, especially with the economy of goat-sheep breeding, people with intermediate up to big in size exploitations use relatively big extent of privately-owned land for the needs of their flocks in pasturage. This fact, in combination with the increased needs in human work of the goat-sheep breeding exploitations on Limnos, lead to the conclusion that the particular cattle-breeders are mainly holders of big goat-sheep breeding exploitations and are exclusively occupied in primary sector and especially in cattle-breeding.

With the second degree factor analysis applied afterwards, the above structures and relations of the factors were even more documented. Specifically, in this analysis the 5 initial factors were used as variables. 
The results that came out of the second degree application of the method are presented in Table 2.

From the second degree factor analysis two factors resulted. The first is reported as "Multi-activity in primary sector" and the second one as "Local rural products support".

According to the cross-correlation factors of the variables with the first factor P 1.2 "Multi-activity in primary sector", it is realized that the Multi-activated on Limnos related to primary sector, are those who are mainly occupied in primary sector and those who are circumstantially occupied in primary sector.

The Multi-activated with their main occupation in primary sector, are described by the initial factor "mainly occupied in agriculture" and while this factor sketches out the farmer's characteristics and their relation with the main agricultural activity the "other" occupation is not clarified. The possibility of a second occupation is however evident, as their exploitations maybe big in size but are mainly extensional and with limited intensity of work, a fact ensuring time for additional work. Due to their relatively low education and residence in no tourist regions of the island, their second occupation is mostly related to second occupation in primary and secondary sector but also to the operation of various shops (commercial, cafes, etc.) in the villages of the rural region of Limnos.

The Multi-activated with a secondary occupation in primary sector are described by the initial factors "Multi-activated with secondary occupation in the primary sector" and "Young, multi-activated occupied in agriculture". Compendiously, they are the heads, the spouses and the young members of the households, whose main occupation is the one in the sector of services and the level of their education is relatively high. Their occupation in primary sector is imposed by the need for supplementary income, which is indeed ensured in a way so that the economic needs of the multi-activated households are satisfied. Their exploitations are small in size, mainly viniculture or cattle-breeding.

By the cross-correlation factors of the variables with the second factor P 2.2 "Local rural products support" it is realized that the exclusively or mainly occupied in primary sector and the exclusively occupied in cattle-breeding who actively participate in rural cooperative societies, have positive opinion, entrust and support the brand name local qualitative rural products ${ }^{[12]}$. It is justifiable to say that the qualitative local rural products have historical, cultural and economical bonds with the region they are produced, while at the same time they constitute choices of the productive base itself, that is to say the farmers. In consequence, the confidence of the exclusively or mainly occupied in rural sector to the products they produced and produce is legitimate, as they perceive that these determine, up to a point, their income and substantially contribute in the gross product of the island. Moreover, they hope in bigger future profits from these products, as there is space for greater exploitation and conquest of higher prices, while they would difficulty abandon the relative cultures or exploitations because they identify their particular qualitative characteristics with those of their island.

More specifically, the direct relation of cattlebreeders with cheese-making and the various kinds of local cheeses produced imposes the cattle-breeders confidence in the quality of these products. The recognition of the product, they produced and still produce, implies their award and in consequence their acceptance of all the local products in general. Furthermore, the participation of the cattle-breeders who own big exploitations in the rural cooperative societies, in a great scale consequences the recognition of the value of other local rural products, such as the honey and wine.

The fourth initial factor "Young, multi-activated occupied in agriculture" is negatively related to the factor "Local rural products support", a fact that implies that the young people that are circumstantially and many times auxiliary occupied in primary sector, do not have a positive opinion and do not entrust the local rural products. Their main occupation in the tertiary sector, their non-systematic avocation with the familial small exploitations and the lack of experience potentially influence the configuration of their negative opinion regarding these products. Their critical attitude towards the traditional activities and the products resulting from them is characteristic and it is focused in their phrases "fame is all we have in the wine" and "the milk is at the same price at the remainder regions in Greece".

This reaction of the multi-activated young persons, who are occupied in agriculture as well, towards the local rural products also, potentially, emanates from the connection in their thoughts of the production of these products with a not particularly charming way of life and with the backbreaking rural occupation. On the contrary, the farmers which declare this as their main profession and are older in age, which supported and were supported by the local products, are in a way represented by them, while they are also indissolubly connected to their tradition.

\begin{tabular}{ll} 
Appendix: Table of variables names \\
\hline AREA & Region of residence \\
FPOS & Position in the family \\
AGE & Age \\
EDUC & Level of education \\
MUAC & Multi-activated \\
MU13 & Multi-activated occupied in primary and tertiary sector \\
ME1 & Main occupation in primary sector \\
ME3 & Main occupation in tertiary sector \\
ROM & Causes that lead to second occupation \\
INPU & Satisfaction from the overall income \\
AGEA & Extent of cultured agricultural land \\
OWEA & Extent of privately owned rural land \\
\hline
\end{tabular}




\begin{tabular}{|c|c|}
\hline ANEA & Extent of used pasture land \\
\hline COOP & Participation in corporative rural organizations \\
\hline $\mathrm{OPCH}$ & $\begin{array}{l}\text { Opinion concerning the advantages of the cheese as a local } \\
\text { product }\end{array}$ \\
\hline OPWN & Trust in the wine as a local qualitative product \\
\hline OPFU & Trust in an other local qualitative product \\
\hline ORIG & $\begin{array}{l}\text { Intention of adopting a new culture that will bring } \\
\text { economic and environmental gain }\end{array}$ \\
\hline
\end{tabular}

\section{REFERENCES}

1. Chatzitheodoridis, F.X., 1998. Multi-activity and special qualitative products in the frame of the integrated development of the islands: The case of Island Limnos, PhD, Aegean University, Mitilini (in Greek).

2. Coccossis, H., 2001. Sustainable development and tourism in small islands: Some lessons from greece». Anatolia, 12:1

3. Gidarakou, I., 1999. Young women's attitudes towards agriculture and women's new roles in the Greek countryside: A first approach. J. Rural Studies, 15: 2.

4. Samaras, G., D. Damianos, X. Kasimis, A. Moesidis and M. Ntemousis, 1995. The multioccupation in the Rural sector and the Developmental Politic in Greece. Foundation of Mediterrenean Studies and Alexandria Editions, Athens (in Greek).
5. Zakopoulou, E., 1999. Multi-occupied and Agriculture: Towards a new tracing of a Multidimensional Phenomenon». In Kasimis X. Loyloydis L, Countryside Land: The Greek Rural Society in the end of Twentieth Century. EKKE and Publications Plethron, Athens (in Greek).

6. Lamprianidis, L., 2003. Local products as a mean for growth support of the less developed regions of the European. Aechoros, 2: 1 (in Greek).

7. Skouras, D. and A. Vakrou, 2002. Willingness to pay for origin labeled products. Br. Food J., 104: 11.

8. Parrott, N., N. Wilson and J. Mundoch, 2002. Spatialising quality: Regional production and the alternative geography of food. European Urban and Regional Studies, 9: 3.

9. Ilbery, B. and M. Kneafsey, 1999. Niche markets for regional specialty food products in Europe. Environment and Planning, 31: 1.

10. Turner, T., 2005. Article 1143, Economist Agricultural Wages and employment.

11. Baldascini, A., 2004. Lead Consultant, GCP/INT/886/FRA, Promoting and Protecting Local Products as a means for sustainable development.

12. Sicco van Gelder, 2003. Global Brand Strategy. http://www.beyond-branding.com/Globprel.pdf 